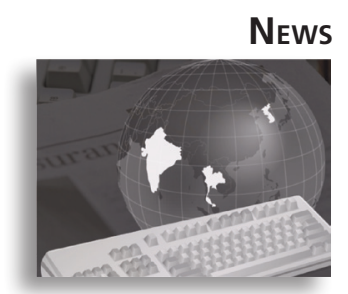

Institutional Profile

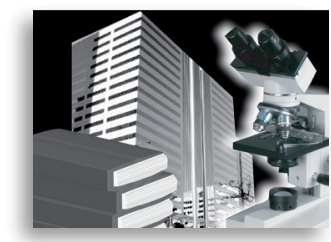

Conference Scene

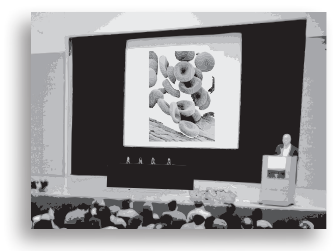

\title{
Biomarkers may help predict risk of Alzheimer's disease
}

\section{A recent study has demonstrated that several cerebrospinal fluid biomarkers show strong accuracy in identifying individuals suffering from mild cognitive impairment who progress to Alzheimer's disease}

Biochemical alterations in the brain are reflected in cerebrospinal fluid (CSF) and substantial efforts have been made to develop biomarkers for the pathogenic processes in Alzheimer's disease (AD) that can be used as diagnostic tools.

"Treatments would need to be initiated very early in the disease process, before the neurodegenerative process is too severe. Much focus has thus been directed on patients with mild cognitive impairment (MCI), which is a syndrome characterized by cognitive impairment beyond the ageadjusted norm, but not severe enough to fulfil the criteria for dementia," explained the authors of the study.

Previous research has demonstrated that CSF biomarkers may be useful for identifying the beginnings of $\mathrm{AD}$ in patients who suffer from MCI. However, the majority of the studies were conducted at single centers.

The recent work, conducted by Niklas Mattsson of the Sahlgrenska Academy (University of Gothenburg, Mölndal, Sweden) and colleagues, was a multicenter study to assess the diagnostic accuracy of the CSF biomarkers $\beta$-amyloid 1-42 (A $\beta 42)$, total tau protein (T-tau) and tau phosphorylated at position threonine 181 (P-tau) in identifying the beginnings of $\mathrm{AD}$ in a much larger group of individuals with MCI.

A total of 750 patients with MCI, 529 with $\mathrm{AD}$ and 304 controls were enrolled into the study. This was followed by a prospective cohort study with patients who have MCI. A total of 12 centers across the USA and Europe were involved in the study and participants were followed for at least 2 years or until their symptoms had progressed to clinical dementia.
The follow-up resulted in 271 individuals with MCI being diagnosed with $\mathrm{AD}$ and 59 with other dementias. Interestingly, the $A \beta 42$ assay had considerable variability between sites. Those who developed AD had lower median $A \beta 42$, and higher P-tau and T-tau levels than patients with MCI who did not develop $\mathrm{AD}$ during the follow-up.

"We determined, in a large multicenter study, that the CSF biomarkers A $\beta 42$, T-tau and P-tau can be used to predict, with good accuracy, which MCI patients will develop $\mathrm{AD}$, as previously found in smaller studies. This multicenter collaboration avoids several of the risks of biases associated with singlecenter studies by having included substantially more patients than previous studies. Although each memory clinic center followed up its cohorts prospectively and used established clinical criteria, a limitation of the present study is the lack of fully harmonized study protocols for all centers, which might account for some of the intercenter variations that we observed," the authors write.

"Using CSF A $\beta 42$, T-tau and P-tau in memory clinics will result in some falsepositive cases, as well as false-negative cases ... Furthermore, they may be useful in enriching study populations for clinical trials of future disease-modifying AD treatments. Until such treatments become available, however, these tests are not generally appropriate for routine clinical use because it is not currently possible to alter the development of AD."

Source: Mattsson N, Zetterberg H, Hansson O et al.: CSF biomarkers and incipient Alzheimer disease in patients with mild cognitive impairment. JAMA 302(4), 385-393 (2009). 


\section{Novel biomarker predicts response to hepatitis $\mathrm{C}$ treatment}

Researchers at Duke University Medical
Center (Durham, NC, USA) have iden-
tified the first genetic marker that is able
to predict a patient's response to hepati-
tis C treatment. Importantly, this newly
identified biomarker may help to explain
why there are such clear racial disparities
in treatment responses, a well-established
phenomenon whose cause has yet to have
been elucidated.
Hepatitis C-an infectious viral disease of
the liver-is one of the most common infec-
tions worldwide and, once established, the
disease can cause cirrhosis, liver cancer and
death. Typically, the recommended treat-
ment for hepatitis C involves a 48 -week-long
course of interferon in conjunction with the
antiviral drug ribavirin. Unfortunately, the

\section{Potential novel fecal biomarkers for detecting gastrointestinal cancers}

In a preliminary evaluation, the methylation patterns of two gene promoters in fecal DNA have demonstrated promise for the detection of gastric and colorectal cancers. It is hoped these markers from could represent a new and effective noninvasive means to detect gastrointestinal (GI) cancers.

Many individuals are reluctant or unwilling to undergo invasive tests for the detection of GI cancers; therefore, the discovery of nonintrusive means to detect such cancers is a priority for researchers. Owing to the fact that cells, including cancer cells, are sloughed off into the lumen of the GI tract, it is possible to use patients' stool samples to analyze DNA from cells present in their feces, and examine the DNA for the presence of cancer cell biomarkers.

In a recent study, researchers analysed the methylation of the RASSF2 and SFRP2 gene promoters from 788 primary gastric and colorectal tumors, aiming to determine whether the methylation patterns of these promoters could act as biomarkers for GI tumorigenesis. The results of the study demonstrated that extensive methylation at these two gene promoters was more likely to be found in GI tumors than in normal tissue.

Noninvasive detection of GI neoplasia through the use of reliable cancer biomarkers may help to identify the cancers earlier and, thus, contribute to reducing the mortality from GI cancers. "By identifying disease-specific methylation patterns for human fecal DNA from advanced gastric and colorectal tumors, we could more accurately identify subjects at high risk for developing, or having developed, advanced tumors."

man am a

Source: Nagasaka T, Tanaka N, Cullings HM et al.: Analysis of fecal DNA methylation to detect gastrointestinal neoplasia. J. Natl Cancer Inst. doi: 10.1093/jncildjp265 (2009) (Epub ahead of print). side effects of treatment can be extremely severe, and not all patients are cured. Of note, it has long been established that African-American patients are less likely to respond to treatment than Caucasians, and that East Asian patients respond the best; however, until now, it has not been known why these racial differences exist.

"For geneticists, understanding response to treatment for hepatitis $\mathrm{C}$ infection has been almost like a Holy Grail," says David Goldstein, senior author of the study.

Now, the discovery of a new marker - a single letter change in which a $\mathrm{T}$ is substituted for a $\mathrm{C}$ in a small segment of DNA near the IL28B gene - may explain a large part of these differences.

Goldstein and his team, studying 1671 hepatitis C patients, found that those patients with the aforementioned single-letter change in their DNA were significantly more likely to respond to treatment than those without it. As the beneficial genotype occurs more frequently in Caucasians than it does in AfricanAmerican individuals, the researchers believe their finding may explain much of the difference in the response rates between these groups of individuals.

In their study, $80 \%$ of those with the favorable genotype eradicated the virus, whereas just $30 \%$ of those with the less favorable genotype were able to do so. Indeed, not least owing to the potential severity of the side effects of treatment, this novel biomarker may help to guide treatment decisions in the future. "The side effects of hepatitis treatment can be brutal, and about half the time, the treatment fails to eradicate the virus. This discovery enables us to give patients valuable information that will help them and their doctors decide what is best for them. This is what personalized medicine is all about," said Goldstein.

Source: Ge D, Fellay J, Thompson AJ et al.: Genetic variation in IL28B predicts hepatitis C treatment-induced viral clearance. Nature doi: 10.1038/nature08309 (2009) (Epub ahead of print). 


\section{Prion identified as a biomarker for pancreatic cancer}

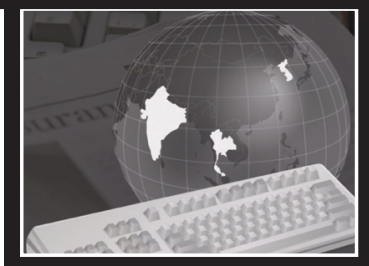

Researchers have identified, for the first time, the prion as a biomarker for pancreatic cancer, according to a study from Case Western Reserve University School of Medicine (Cleveland, OH, USA). Importantly, the research may pave the way for new therapeutic approaches to treat this deadly cancer.

Prions are infective proteins, the accumulation of which in the brain of an individual is established to cause new variant Creutzfeldt-Jakob disease, the human form of bovine spongiform encephalopathy, more commonly known as mad cow disease. Outside of the brain, however, little is known about prions.

Now, researchers have identified the mechanism by which prions cause tumors to grow more aggressively. A new study reports that in human pancreatic cancer cells, the prion is incompletely processed and binds to a molecule inside the cell known as filamin A. The binding of the incompletely processed prion to filamin A disrupts the cell's cytoskeleton and signaling, causing the tumor cell to grow more aggressively.

Importantly, the team behind the new study found that those pancreatic cancer patients who had incompletely processed prion proteins in their pancreatic cancer cells have significantly shorter survival compared with those patients whose tumor cells did not contain prions.

"Currently there is no early diagnostic marker for pancreatic cancer. Detection of the incompletely processed prion may provide such a marker. Preventing the binding of prion to filamin A may open new avenues for therapeutic intervention of this deadly disease" says Chaoyang $\mathrm{Li}$, one of the study's authors.

Source: Li C, Yu S, Nakamura F et al.: Binding of pro-prion to filamin A disrupts cytoskeleton and correlates with poor prognosis in pancreatic cancer. J. Clin. Invest. 119(9), 2725-2736 (2009).

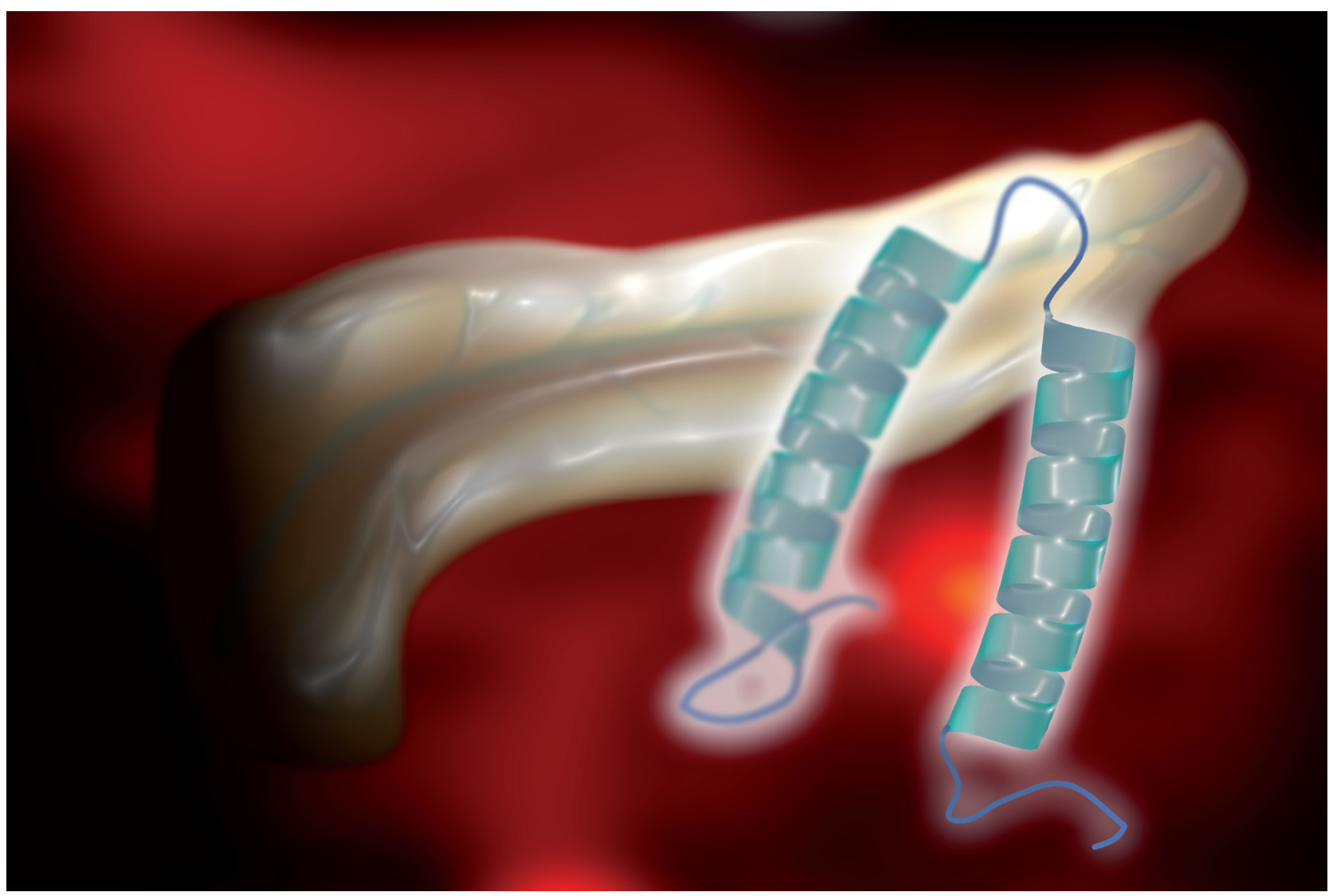

